\title{
L'enseignement à distance à l'Université au Niger : un processus en construction
}

\author{
Moussa Mohamed Sagayar, Université Abdou Moumouni
}

\section{DOI : 10.51186/journals/ed.2020.10-2.e349}

\section{Résumé}

Cet article étudie le développement de l'enseignement à distance à l'Université Abdou Moumouni (UAM). Nous relevons que, malgré leur pertinence, l'enseignement à distance et le développement des technologies éducatives ne sont pas ou peu développées à l'UAM. Sur la base de ce constat, nous avons procédé à une analyse systématique des travaux en situant l'état du savoir dans le domaine sur la base d'un questionnement à l'origine de notre revue de littérature : l'exploitation du potentiel des Technologies de l'Information et de la Communication (TIC) et l'enseignement à distance peuvent-ils créer une nouvelle culture de l'apprentissage ? Nous poursuivons un double objectif : le premier consiste à analyser l'enseignement à distance et son processus de construction, et le second se propose d'examiner les possibilités d'apprentissage à distance en déterminant leurs apports réels dans les changements des pratiques des enseignants. Les principaux résultats montrent le peu d'expertise de l'UAM et recommandent la formation des enseignant-es et des étudiant-es en matière de technologies éducatives pour développer l'enseignement à distance.

Mots-clés : enseignement à distance, technologie de l'information et de la communication, technologies éducatives, pratiques d'enseignement, ressources pédagogiques

\section{Abstract}

This paper studies the development of distance education at Abdou Moumouni University (UAM). Despite their relevance, distance education and educational technologies remain undeveloped or underdeveloped at UAM. Based on this observation, we carried out a systematic analysis of research in this field based on the question that motivated our literature review, namely: can tapping into the potential of Information and Communication Technologies (ICT) and distance education create a new culture of learning? The study has two aims: first, to analyse distance education and the process by which it is constructed; and second, to examine the possible effects of distance learning by determining how it actually changes teachers' practices. The main results show the limited expertise of UAM and recommend the training of teachers and students in educational technologies to develop distance education. 
Keywords: distance education, technologies information and communication, educational technologies, teaching practices, teaching resources

\section{INTRODUCTION}

Dans les universités africaines en général, l'enseignement à distance est un enjeu de développement. Même si les situations sont diverses selon les sous-régions et les pays, l'enseignement à distance en Afrique subsaharienne, et spécifiquement dans sa partie francophone, reste en général bien en-dessous des attentes. Ces attentes exprimées tant en termes de quantité que de qualité donnent lieu à des bilans mitigés quoique différents.

L'Afrique, à l'instar de toutes les régions du monde, a, en prélude à la conférence d'Incheon, opéré un bilan de son système éducatif au regard des objectifs mondialement fixés pour 2015, en vue des projections et de l'élaboration du plan d'actions de l'agenda 2030. Les chefs d'État africains prenaient l'engagement suivant :

Nous prenons enfin l'engagement de renforcer la science, la technologie et l'innovation. Les technologies de l'information et de la communication (TIC) doivent être mises à profit pour renforcer les systèmes éducatifs, la diffusion du savoir, l'accès à l'information, ainsi que l'efficacité et la qualité de l'apprentissage, et assurer une offre de services plus performante. (UNESCO, 2016, p. 8)

Les TIC ont montré qu'il est possible d'améliorer les pratiques pédagogiques par de nouveaux modes d'enseignement et de nouvelles formes d'apprentissage. Les enseignants peuvent développer l'usage des TIC dans les enseignements en créant des ressources numériques pédagogiques de qualité.

S'agissant de l'enseignement à distance, objet de notre travail, de nombreuses recherches ont montré des évolutions numériques et des mutations dans la construction de savoir en ligne. On passe d'une modalité d'apprentissage pour laquelle les étudiant-es sont dans une posture basse qui les oblige à suivre des cours programmés (cours magistraux, travaux dirigés, travaux pratiques) dans un emploi du temps soumis à la disponibilité des salles et de l'enseignant-e, à des apprentissages à distance pour lesquels l'organisation des enseignements est flexible et ouverte. Une dynamique des TIC se met en place progressivement au niveau de l'enseignement supérieur en Afrique à travers des objectifs bien précis :

L'utilisation de l'EAD et des TIC pour élargir l'accès à l'enseignement supérieur en Afrique a deux objectifs : augmenter les effectifs et offrir davantage de possibilités aux étudiant-es qui ne peuvent aller à l'université parce qu'ils habitent trop loin des campus ou que leurs horaires de travail ne leur permettent pas de s'y rendre à l'heure des cours. (Murphy, et al., 2002, p. 21) 
Cette dynamique est confrontée à de sérieux problèmes, notamment au manque de compétences techniques des enseignant-es et des étudiant-es d'une part, et au manque de réflexion profonde sur comment les TIC peuvent aider les systèmes éducatifs à remplir leur mission d'autre part (Attenoukon, 2011).

Akouété-Hounsinou (2012) explore une autre voie de l'intégration des TIC en Afrique, mais cette fois-ci sous la double dimension de leur utilisation et de la mise en place de programmes de formation à distance des enseignant-es. Selon lui, « le recours aux TIC dans l'éducation à distance constitue une façon de dispenser un enseignement de haute qualité » (pp. 147-148).

L'enseignement à distance devient un paradigme se focalisant sur le changement des pratiques enseignantes et l'amélioration de l'apprentissage des étudiant-es du supérieur pour développer de nouvelles compétences (Karsenti, 2009). Ainsi, l'enseignant-e prépare ses enseignements à partir de supports pédagogiques et des informations qui nécessitent une forte implication des étudiant-es. Ces derniers/ères s'engagent dans un processus de sélection de ces informations utiles pour leur apprentissage, et s'organisent en conséquence pour accéder aux savoirs construits à partir d'un scénario pédagogique et d'un environnement technologique (Chan, et al., 2019).

Ainsi, en nous inscrivant dans la dynamique de l'environnement d'apprentissage à distance, nous montrons dans une première partie en quoi l'exploitation du potentiel des Technologies de l'Information et de la Communication pour l'Enseignement (TICE) peut créer une nouvelle culture de l'enseignement à distance. Dans une deuxième partie, nous décrivons le processus de mise en place de l'enseignement à distance en termes d'environnement d'enseignement et d'apprentissage en ligne dans lequel enseignant-es et étudiant-es peuvent interagir, communiquer, discuter, et utiliser des ressources numériques pédagogiques. La troisième partie va être consacrée à la présentation d'un Master en Science de l'Éducation comme un exemple d'aboutissement d'un projet d'étude à distance à l'Université Abdou Moumouni (UAM).

\section{DÉVELOPPEMENT DE L'ENSEIGNEMENT À DISTANCE ET APPORT REEL}

Les technologies de l'enseignement à distance se développent et prennent place à l'université. II s'agit d'un mode d'enseignement qui utilise les outils numériques et les moyens de l'internet pour l'enseignement à distance (Murphy, et al., 2002). Ces auteur-es situent les avancées technologiques et précisent :

Presque tous les pays africains ont au moins un programme d'enseignement supérieur à distance. Ces programmes visent à accroître l'accès et concernent les étudiants qui ne peuvent venir sur le campus des universités, soit qu'ils habitent trop loin soit que leurs occupations professionnelles les empêchent d'assister aux cours. (p. ix) 
Les TIC ont joué un rôle moteur dans le développement de l'enseignement à distance, en offrant des possibilités techniques via le Web en mode hybride.

\subsection{Vers la création d'une nouvelle culture de l'apprentissage à distance au supérieur}

L'enseignement supérieur est un secteur qui assure l'accès de tous et toutes à un enseignement de qualité. La problématique de la qualité des enseignements/apprentissages est ainsi explicitement identifiée comme une priorité, tout comme celle de l'apprentissage tout au long de la vie qui prend en compte de nouvelles postures de l'enseignant-e et de nouvelles formes d'apprentissage, le tout en ligne. L'enseignement supérieur est donc reconnu comme étant un levier important pour développer l'enseignement à distance, notamment en Afrique de l'Ouest. Ce rôle moteur implique cependant une évolution des systèmes d'enseignement supérieur existants pour leur permettre de mieux répondre aux possibilités d'accès aux savoirs et aux informations exceptionnelles à travers l'usage des TICE. L'enseignement à distance modifie les pratiques enseignantes, et prend en considération les caractéristiques des apprenant-es et de l'espace d'apprentissage en statuant sur l'efficacité technique et pédagogique du dispositif mis en place (Ferone \& Lavenka, 2015).

L'élaboration d'un diagnostic approfondi et intégré des potentialités de développement de l'enseignement à distance est indispensable pour la mise en place d'un dispositif d'enseignement à distance à l'UAM. Cette étude diagnostique doit aboutir à une analyse de viabilité et de pérennisation du dispositif technique, la mise en place d'une ingénierie pédagogique et l'élaboration d'un plan de développement et sa stratégie d'opérationnalisation. C'est bien le développement de l'enseignement à distance pour favoriser le développement du numérique éducatif en tant que complément ou alternative à un apprentissage innovant et ouvert qui est recherché. Ce type d'apprentissage offre des possibilités pour les bénéficiaires (enseignant-es-chercheur-es et étudiant-es) de s'investir dans un programme d'échanges et de productions de ressources numériques pédagogiques à travers des applications virtuelles interactives, des contenus en format numérique ou une mise en relation entre pairs (Pairagogie ${ }^{1}$, usage collectif et/ou collaboratif).

En 2015 une étude de Ferone \& Lavenka a démontré que l'enseignement à distance est « une modalité technico-pédagogique de formation à distance qui permet à des personnes d'établir des échanges synchrones pouvant utiliser l'image, le son et le texte » (p. 3). Ces auteur-es expliquent que l'enseignement à distance est apparu dans l'enseignement supérieur, avant de gagner le secondaire et le primaire au fur et à mesure que les technologies numériques se sont développées pour s'adapter à une demande sociale et économique qui évolue rapidement afin de construire des stratégies et plans opérationnels. Ces ambitions de qualité et de durabilité

\footnotetext{
${ }^{1}$ Le précurseur de la pairagogie (peeragogy), Howard Rheingold en 2012 a initié le processus d'apprentissage collectif de groupes d'individu-es en ligne autour d'un savoir-faire sans la présence d'un-e enseignant-e. L'apprentissage ne se fait pas uniquement au contact de l'enseignant-e, les apprenant-es apprennent également au contact de ses pairs. La pairagogie favorise l'apprentissage par les pairs d'un savoir utile et insiste sur la façon dont les personnes apprennent ensemble sans enseignant-e.
} 
clairement affichées par les universités en général, et l'UAM en particulier, constituent un défi pour l'Afrique subsaharienne qui est une des régions du monde où la situation de l'enseignement supérieur est préoccupante.

\subsection{Enseignement à distance : quelles perspectives pour l'UAM ?}

De nombreuses études font un point de situation sur le contexte de la Formation Ouverte et $\grave{A}$ Distance (FOAD) en Afrique de l'Ouest Francophone. Elles situent l'enseignement à distance comme une nouvelle attraction du fait de ses modes d'apprentissage et de son expansion dans le supérieur (Tonye, 2013). On peut voir également voir la place qu'occupe l'enseignement à distance dans les politiques étatiques et les stratégies institutionnelles universitaires autour de leurs potentialités, les modalités de mise en œuvre, leur ancrage institutionnel et les postures des enseignants (Béché, 2016).

Sur un tout autre plan, l'enseignement à distance mobilise de nombreux partenariats et accords-cadres pour soutenir les projets structurants qui se mettent en place entre plusieurs universités à travers un consortium (Mohamed Sagayar, 2018). Ces projets dits structurants ont la particularité d'organiser les universités autour d'un idéal commun qui aboutit à un travail collaboratif sur la gouvernance, le pilotage, la coordination du projet, la stratégie de déploiement, la pérennisation du projet et son modèle économique.

L'intégration des TIC est également abordée pour montrer son indispensable lien avec le développement de l'enseignement à distance. Attenoukon (2020) analyse la dimension intégrative des TIC dans les pratiques pédagogiques des enseignant-es et leurs liens possibles avec l'enseignement à distance. II en déduit que les TIC sont dans le contexte universitaire une source de développement des compétences professionnelles des enseignant-es et des pratiques d'apprentissage des étudiant-es.

Les TIC sont étudiées à travers leurs usages éducatifs dans les pratiques professorales (Ouedrago, 2020). Les enseignant-es dans leurs usages développent des compétences techniques et pédagogiques avec une approche centrée sur l'acceptation des TIC comme un outil au service du développement de l'enseignement supérieur.

La place de l'enseignement à distance chez les auteur-es africain-es oriente les réflexions sur les pratiques pédagogiques en lien avec l'intégration des TIC et leurs usages dans des contextes universitaires en pleine mutation tant sur le plan pédagogique que sur le plan didactique. Les thématiques abordées questionnent principalement l'appropriation du potentiel des technologies éducatives par les enseignant-es et les apprenant-es de l'enseignement primaire, secondaire et universitaire.

Dans un contexte de développement des technologies numériques, comment faire de l'enseignement à distance une réalité à I'UAM ? Passer de l'enseignement en présentiel et 
envisager la mise en place d'un enseignement à distance des pratiques enseignantes et des modalités d'apprentissage peut paraître une mission périlleuse, voire impossible. C'est du moins un constat que l'on peut faire au risque de se tromper sur les véritables enjeux de l'enseignement à distance et son arsenal technico-pédagogique qui l'accompagne.

L'enseignement à distance offre d'une part, des garanties de développement des enseignements/apprentissages en prenant en compte la question de la massification des effectifs. Et d'autre part, il montre des limites d'ordres pédagogique et technique. On peut supposer que des transformations de pratiques plus créatives et plus collaboratives peuvent ainsi voir le jour, mais il est important de mettre en place un dispositif technique, fiable et pérenne. Ainsi mettre des technologies à disposition des enseignant-es-chercheur-es et des étudiant-es permet aux usages de se développer (Bruillard, 2010). Les apports des technologies dans une perspective de rénovation pédagogique et de modernisation institutionnelle sont aujourd'hui nécessaires pour le développement des systèmes d'enseignement supérieur au niveau mondial (Ferone, 2011).

À l'UAM, l'enseignement à distance a fait l'objet d'une première réflexion en 2015 autour des potentialités de développement de la formation à distance et de la formation numérique. À l'origine de cette réflexion, il était question d'étudier la possibilité d'un partenariat avec l'Agence Universitaire de la Francophonie (AUF) pour développer le numérique éducatif à travers les FOAD. Pour l'UAM, il fallait examiner comment mettre en place un plan de développement de l'enseignement à distance qui soit techniquement, pédagogiquement et organisationnellement faisable et viable. Mais aussi, voir si les enseignant-es-chercheur-es étaient prêt-es à s'engager dans une innovation qui va naturellement constituer une véritable et profonde mutation dans les pratiques enseignantes intégrant les TIC pour apprendre. II y a donc de véritables enjeux pour s'investir dans un programme innovant de développement de l'enseignement à distance et de répondre à la massification de l'enseignement, puisque les effectifs d'étudiant-es se sont considérablement accrus à l'UAM, passant de 7722 en 2006-2007 à 16295 en 2014-2015.

Au problème de la massification, s'ajoute également l'absence d'infrastructures, d'équipements, de connexion internet et de ressources humaines compétentes. La volonté de modernisation de l'UAM s'inscrit dans une perspective de développement du numérique et de ses activités connexes. Cette volonté institutionnelle se traduit par un besoin d'innover de certains enseignant-es-chercheur-es. En effet, elles/ils sont au cours de ces dernières années près de 200 enseignant-es et le personnel de l'Université à être formé-es par l'AUF à travers le Campus numérique francophone de Niamey. II y a donc un vivier favorable d'enseignant-es initié-es à la FOAD. Ces derniers/ères sont aptes à former leurs collègues et à encadrer le développement de dispositifs de formation utilisant les technologies.

Mais pour l'UAM, mettre en place un dispositif de formation nécessite de penser une stratégie pour identifier les ressources pédagogiques numériques porteuses de contenu spécifique au développement de l'enseignement à distance. On le voit bien, l'enseignement à distance et le 
développement du numérique, même s'ils sont susceptibles d'apporter des réponses concrètes aux difficultés de l'enseignement supérieur, méritent une attention particulière.

L'enseignement à distance fait débat dans la communauté scientifique en ce qu'il est un facteur de développement qui permet à un groupe d'individu-es de rester en contact et en ligne (Jézégou, 2010). Les acteurs/trices avertis du domaine pensent que l'enseignement à distance, offre de la qualité dans les apprentissages en restant accessible et ouvert (Power, 2002). Certain-es de ses acteurs/trices pensent qu'il y a des limites structurelles et institutionnelles au développement et à la promotion de ce type d'enseignement (Jézégou, 2008). D'autres auteur-es soulignent aussi d'importants problèmes techniques limitant le bon fonctionnement des systèmes d'apprentissage (Ferone \& Lawenka, 2015). Dans le cadre d'une étude diagnostique menée à l'UAM en 2015 portant sur l'appui au développement de la formation à distance et du numérique éducatif à l'Université, il ressort que les enseignant-es sont confronté-es à des difficultés d'ordre pédagogique quand elles/ils décident de s'investir dans les TICE. L'étude a révélé que la connexion internet n'était pas du tout stable, les infrastructures sont insuffisantes devant les besoins des différent-es utilisateurs/trices. Une autre étude menée en 2017 (sur le diagnostic des pratiques, besoins et attentes des usagers) a révélé aussi quelques faiblesses : les plus dommageables sont d'ordre organisationnel (on relèvera un déficit d'échanges d'expérience entre les universités dans les domaines technique, pédagogique et de gestion).

\subsection{Des opportunités pour réussir la transition de l'UAM vers l'enseignement à distance}

Une certaine timidité apparait dans l'usage pédagogique de ressources, et une dispersion des efforts déployés pour exploiter dans un cadre global de gouvernance du numérique des ressources numériques pédagogiques qui foisonnent en ligne (Mohamed Sagayar, 2018). En revanche, on note des opportunités qui peuvent être exploitées pour mieux réussir la transition vers l'enseignement à distance. Ces opportunités permettent à l'UAM d'intégrer le numérique comme un enjeu stratégique pour développer, innover et prendre des initiatives dans le seul but d'exploiter les possibilités offertes par les TIC, pour mieux diffuser et partager les savoirs pédagogiques auprès des enseignant-es.

Aujourd'hui, sur internet, des dizaines de milliers de cours en ligne existent. A l'UAM, les enseignant-es et les étudiant-es recherchent, sans méthode, en se contentant de passer par Google. Or, il existe une offre structurée, libre de droits, élaborée par les universités françaises réunies en consortium dans des " universités numériques thématiques » (UNT), qu'il est possible de rendre disponible pour les enseignant-es et étudiant-es nigériens. Les Ressources Éducatives Libres (REL) de droits sont disponibles aujourd'hui sur internet. C'est une occasion probante pour exploiter ce potentiel des REL et créer une nouvelle culture de l'apprentissage à I'UAM. Pour permettre une véritable appropriation du potentiel des REL dans le cadre du développement des savoirs autochtones, elles peuvent être utilisées pour soutenir le « principe de participation » commun dans de nombreuses communautés d'origine, ainsi que la 
préservation et la diffusion du savoir autochtone qui a traditionnellement été ouvert. Les REL en Afrique peuvent permettre un meilleur accès à l'apprentissage, en augmentant la diversité du matériel pédagogique, la réduction des coûts pour les institutions (reproduction libre par exemple).

Ce qui précède est un ensemble de possibilités pour booster l'enseignement à distance parce qu'il impacte l'ensemble des activités et modes de fonctionnement de l'UAM. Bien au-delà des effets de mode, l'UAM se trouve face au défi de maîtriser son impact en construisant la cohérence de son évolution numérique plutôt que de le subir.

\section{PROCESSUS DE MISE EN PLACE DE L'ENSEIGNEMENT À DISTANCE À L'UAM : DE NOUVELLES REPRESENTATIONS À CONSTRUIRE}

Cette partie s'intéresse au développement de la formation à distance à l'UAM et à son apport réel dans l'amélioration des pratiques enseignantes. Elle se propose de présenter une étude de cas sur la création d'un Master en Sciences de l'Éducation, un projet qui répond à un triple enjeu : atteindre les apprenant-es vivant loin des campus, réduire les coûts de fonctionnement et de séjours des apprenant-es, et faciliter l'accès aux apprenant-es ne pouvant pas quitter leur pays ou leur emploi pour résider et suivre les cours en présentiel à Niamey.

\subsection{L'enseignement à distance à l'UAM : vers une ébauche de solution}

À l'UAM, les enseignements en présentiel ne sont pas toujours perçus à leur juste valeur par les étudiant-es. Dans la pratique, il y a de nombreux problèmes liés aux infrastructures et aux équipements. Les pratiques d'enseignement-apprentissage se déroulent souvent avec d'énormes difficultés pour la mise en œuvre des formes d'enseignement classiques. Les étudiant-es font face à des contraintes d'ordre psychologique (attention et motivation ne s'articulent pas).

Les enseignant-es font face à un problème d'ingénierie pédagogique et didactique (Sensevy, 2018) pour enseigner les grands groupes. Tous ces problèmes rendent difficile la construction du savoir lors de la réalisation des Cours Magistraux (CM), la réalisation des Travaux Dirigés (TD) et des Travaux Pratiques (TP) en présentiel. Quelles préconisations pour aller vers une solution qui pourrait intégrer une stratégie pédagogique et réaliser les cours à distance, ou en semi présentiel en fonction des ordres d'enseignement ? L'UAM s'organise pour mettre en place une stratégie de développement de l'éducation numérique. Avec l'expansion de l'enseignement à distance dans le supérieur liée au développement technologique, I'UAM entend promouvoir l'enseignement à distance, en ce qu'il est porteur d'espoir, car accessible à un grand nombre. 


\subsection{Une volonté institutionnelle et académique, mais une absence de culture numérique chez les enseignant-es et les étudiant-es}

L'UAM a la volonté de développer la FOAD et les TICE pour permettre aux enseignanteschercheur-es d'innover. Mais cette volonté institutionnelle est marquée par une absence de culture numérique chez les enseignant-es et chez les étudiant-es.

Le Campus numérique francophone a organisé de très nombreux ateliers de formation à distance (environ 200 enseignant-es et le personnel de l'Université formé-es), mais force est de constater qu'à l'heure actuelle, seuls 6 enseignant-es sur 395 ont réellement déposé leurs cours sur une plateforme de formation à distance mise à leur disposition.

Au niveau de la gouvernance et de l'organisation des TIC à I'UAM, les moyens informatiques et les usages pédagogiques des technologies sont une composante unique, ce qui laisse entrevoir une confusion dans la gestion de l'informatique (aspects techniques) et la pédagogie (usage et ingénierie pédagogique). II est donc important de procéder à une séparation des deux composantes pour envisager une intégration judicieuse des TIC à la pédagogie. Si cette articulation est faite, on pourra ainsi étudier la possibilité de développer la FOAD et des TICE.

À l'UAM, les filières spécialisées en informatique sont très peu développées. La faculté des Sciences offre des formations en TIC, mais très orientées vers les mathématiques. Les étudiant-es comme les enseignant-es font recours à l'informatique pour des usages classiques (la bureautique : traitement et fonctionnalités avancées).

\subsection{Un schéma directeur de la formation à distance à l'UAM : quelques suggestions et orientations des acteurs/trices interrogé-es}

Le numérique est stratégique parce qu'il impacte l'ensemble des activités et modes de fonctionnement des universités. Bien au-delà des effets de mode, I'UAM se trouve face au défi de maîtriser son impact en construisant la cohérence de son évolution numérique plutôt que de le subir.

Dans le cadre d'un diagnostic des pratiques et usages du numérique à l'université, nous avons interrogé les principales/aux acteurs/trices (recteurs, vice-recteurs, secrétaires généraux, enseignant-es-chercheurs, étudiant-es et technicien-nes informatiques) sur les conditions qui pourraient favoriser le développement de l'enseignement à distance. Ces avis personnels portent sur la gouvernance du numérique pour les recteurs, la formation et l'accompagnement des enseignant-es pour les vice-recteurs, les textes réglementaires pour les secrétaires généraux, l'ingénierie pédagogique pour les enseignant-es, l'organisation et la gestion des enseignements pour les étudiant-es, et les aspects techniques pour les informaticien-nes. Les réponses offrent quelques pistes intéressantes réunies dans le tableau ci-dessous. 
Tableau 1. Réponses des acteurs/trices

\begin{tabular}{|c|c|c|}
\hline Acteurs & Questionnements & Synthèses réponses \\
\hline Recteurs & $\begin{array}{l}\text { Quelle organisation des } \mathrm{TIC} \text { et de } \\
\text { l'enseignement à distance à } \\
\text { l'Université ? }\end{array}$ & $\begin{array}{l}\text { Instituer et développer les TIC et } \\
\text { l'enseignement à distance dans une } \\
\text { perspective intégration des } \\
\text { technologies éducatives aux } \\
\text { pratiques pédagogiques }\end{array}$ \\
\hline Vice-Recteurs & $\begin{array}{l}\text { Comment accompagner les enseignant- } \\
\text { es dans les pratiques et usages des } \\
\text { ressources numériques? }\end{array}$ & $\begin{array}{l}\text { Accompagner les enseignant-es } \\
\text { dans l'exploitation pédagogiques et } \\
\text { didactiques des TIC dans leurs } \\
\text { enseignements }\end{array}$ \\
\hline $\begin{array}{l}\text { Secrétaires } \\
\text { généraux }\end{array}$ & $\begin{array}{l}\text { Comment intégrer le numérique éducatif } \\
\text { dans les pratiques pédagogiques? }\end{array}$ & $\begin{array}{l}\text { Mettre en place un cadre } \\
\text { réglementaire qui donne des } \\
\text { orientations technopédagogiques } \\
\text { pour inciter les enseignant-es à } \\
\text { adhérer à une telle innovation } \\
\text { pédagogique }\end{array}$ \\
\hline $\begin{array}{l}\text { Enseignant-es- } \\
\text { chercheur-es }\end{array}$ & $\begin{array}{l}\text { Quels apports des TIC dans le } \\
\text { développement de l'enseignement à } \\
\text { distance? }\end{array}$ & $\begin{array}{l}\text { Changer les postures et les } \\
\text { pratiques pédagogiques } \\
\text { Former à la pédagogie universitaire } \\
\text { numérique } \\
\text { Intégrer les TIC à la pédagogie }\end{array}$ \\
\hline Étudiant-es & $\begin{array}{l}\text { L'enseignement à distance peut-il être } \\
\text { une stratégie pour améliorer vos } \\
\text { apprentissages et une réponse aux } \\
\text { enjeux de la massification des effectifs? }\end{array}$ & $\begin{array}{l}\text { Résoudre les problèmes liés aux } \\
\text { manques de salles de cours et des } \\
\text { équipements informatiques } \\
\text { Favoriser de nouvelles postures } \\
\text { dans les pratiques d'apprentissage }\end{array}$ \\
\hline Informaticien-nes & $\begin{array}{l}\text { L'enseignement à distance nécessite la } \\
\text { mise en place d'un dispositif technique, } \\
\text { quelles précautions prendre pour un } \\
\text { fonctionnement effectif? }\end{array}$ & $\begin{array}{l}\text { Disposer d'une connexion Internet } \\
\text { permanente haut débit (20 Mbit/s) } \\
\text { Disposer d'une plateforme Moodle } \\
\text { fonctionnel }\end{array}$ \\
\hline
\end{tabular}

Ces réponses offrent quelques pistes intéressantes pour la mise en place un schéma directeur de l'enseignement à distance qui est présenté dans le tableau suivant : 
Tableau 2. Schéma directeur de l'enseignement à distance

\begin{tabular}{|c|c|c|c|c|c|}
\hline Étape 1 & Étape 2 & Étape 3 & Étape 4 & Étape 5 & Étape 6 \\
\hline $\begin{array}{l}\text { Gouvernance } \\
\text { du numérique } \\
\text { pour instituer } \\
\text { et développer } \\
\text { l'enseignement } \\
\text { à distance }\end{array}$ & $\begin{array}{l}\text { Intégration des } \\
\text { TIC dans les } \\
\text { pratiques } \\
\text { pédagogiques }\end{array}$ & $\begin{array}{l}\text { Mise en place } \\
\text { d'un cadre } \\
\text { réglementaire } \\
\text { pour } \\
\text { développer } \\
\text { l'enseignement } \\
\text { à distance }\end{array}$ & $\begin{array}{c}\text { Formation des } \\
\text { enseignant-es } \\
\text { à la pédagogie } \\
\text { universitaire et } \\
\text { à l'intégration } \\
\text { des TIC dans } \\
\text { les pratiques } \\
\text { pédagogiques }\end{array}$ & $\begin{array}{l}\text { Formation des } \\
\text { étudiant-es } \\
\text { aux } \\
\text { fonctionnalités } \\
\text { d'une } \\
\text { plateforme de } \\
\text { cours en ligne }\end{array}$ & $\begin{array}{c}\text { Infrastructures } \\
\text { et } \\
\text { équipements } \\
\text { avec une } \\
\text { connexion } \\
\text { Internet haut } \\
\text { débit } \\
\text { Plateforme } \\
\text { Moodle } \\
\text { fonctionnel }\end{array}$ \\
\hline
\end{tabular}

\section{UNE ÉTUDE CAS : UN MASTER EN SCIENCES DE L'ÉDUCATION EN PRÉSENTIEL ET À DISTANCE}

Le Master Professionnel Ingénierie en Conception et Gestion des Alternatives Educatives (MICGAE) sous régional ${ }^{2}$ a été créé dans le cadre de la mise en place d'un projet de plateforme d'enseignement à distance à l'Ecole Normale Supérieure de l'Université Abdou Moumouni de Niamey. II s'inscrit dans le Programme Régional Interinstitutionnel pour la Qualité de l'Éducation (PRIQUE).

Le processus de formulation du Master a réuni des experts de cinq pays (Bénin, Burkina Faso, Mali, Niger et Tchad) et a abouti à la validation du programme de formation et des textes l'encadrant, par les représentant-es des cinq pays. Le Master est ouvert en Octobre 2017 avec une première cohorte de 23 étudiant-es ${ }^{3}$ (personnels des Ministères, des Organisations Non Gouvernementales (ONG) des Organisations de la Société Civile (OSC) provenant des cinq pays cités précédemment. Cette première cohorte a suivi les cours en présentiel pendant deux ans. La modalité d'enseignement en présentiel a été choisie dès le début du projet avec des ouvertures possibles pour la prochaine cohorte sur le couplage présentiel et distanciel ${ }^{4}$ (nommé ici semi présentiel).

\subsection{Processus de mise en place du Master}

Une étude diagnostique (Akkari \& Soumana, 2014) a permis d'identifier les potentialités de développement de la formation numérique et de la formation à distance, pour répondre aux enjeux du développement technologique, au regard de l'existant et des partenaires intervenant

\footnotetext{
${ }^{2}$ Le Bénin, le Burkina Faso, le Mali, le Niger et le Tchad sont les pays concernés par ce Master accompagné dans sa mise en œuvre par la Coopération Suisse en Afrique de l'Ouest.

${ }^{3}$ Le M-ICGAE compte 23 apprenant-es sur 30 sélectionnés, dont 5 femmes (2 du Niger, 2 du Tchad et 1 du Togo) et 18 hommes (2 du Bénin, 1 du Mali, 2 du Tchad, 2 du Burkina Faso, et 13 du Niger).

${ }^{4}$ Avec la modalité asynchrone, il n'y a pas de créneau commun, les échanges d'informations se déroulent sur des forums ou par e-mail, ce qui ne nécessite pas que tout le monde soit connecté en même temps.
} 
dans le domaine de l'éducation alternative. La figure suivante explique le processus en cinq étapes.

Figure 1. Processus de mise en place du Master

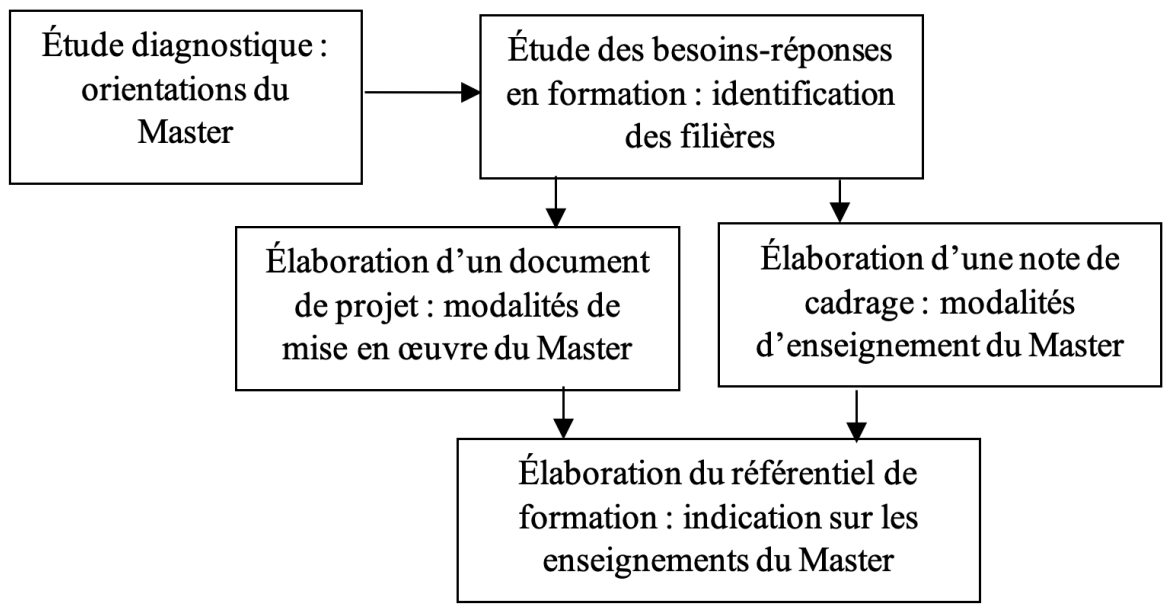

\subsection{Passage du Master en semi présentiel}

II s'avère que la modalité retenue jusqu'à présent (le présentiel) est couteuse en temps, surtout dans le cas du master professionnel qui dure deux années (quatre semestres). Les apprenantes s'absentent de leur poste pour suivre la formation à l'étranger, quand ils ne sont pas nigérien-nes pendant deux années.

Des contraintes sont apparues mettant en cause les cours en présentiel pour des employé-es contractuel-les. II a été envisagé, pour la deuxième phase du Master (2019-2020), de faire les formations en semi présentiel (organisation des cours parfois en présentiel, parfois à distance à partir d'une plateforme). II est prévu des regroupements par semestre sur le site de la formation à Niamey, pour permettre à un plus grand nombre d'apprenant-es d'accéder et de rester à la formation, le reste du temps, les cours sont faits à distance, chaque étudiant-e étant dans son pays.

Dans le cadre du semi-présentiel, il s'est agi de créer une plateforme Moodle de mise en ligne des cours (création d'un espace numérique, hébergement de la plateforme, infrastructures et équipements) avec l'accompagnement du Campus du Nouvel Espace Universitaire Francophone (CNEUF). L'ENS met en place un système autonome tout en ayant son propre serveur et ses ressources matérielles renforcées par la connexion internet du campus. Une formation en ingénierie pédagogique, et l'accompagnement des intervenant-es et des apprenant-es, ont été déployés pour leur permettre d'accéder aux fonctionnalités de la plateforme. II y a eu la révision de la maquette de formation en présentiel en vue d'identifier les Unités d'Enseignement (UE) et les Eléments Constitutif de l'Unité d'Enseignement (ECUE) candidats au semi-présentiel (au moins 70\% des UE/ECUE) en fonction des UE/ECUE à dimension pratique importante difficilement gérables à distance. Après tout, la réorganisation 
des cours a permis de procéder au récapitulatif des semestres suivants les cohortes d'étudiant-es par année académique (Tableau 3).

\section{Tableau 3. Récapitulatif des semestres suivants les cohortes d'étudiant-es par année} académique

\begin{tabular}{|c|c|c|c|c|}
\hline 2017 & 2018 & 2019 & 2020 & 2021 \\
\hline C1S1 (sept) $^{5}$ & C1S2 (fév) & C1S4 (fév) & C2S4 (fév) & C3S4 (fév) \\
\hline- & C1S3 (sept) & C2S2 (fév) & C3S2 (fév) & C4S2 (fév) \\
\hline- & C2S1 (sept) & C2S3 (sept) & C3S3 (sept) & C4S3 (sept) \\
\hline- & - & C3S1 (sept) & C4S1 (sept) & $\begin{array}{c}\text { C4S4 } \\
\text { (fév.2022) }\end{array}$ \\
\hline 1 semestre & 3 semestres & 4 semestres & 4 semestres & 4 semestres \\
\hline
\end{tabular}

À titre indicatif pour les semestres C1S1 et C1S2, il est prévu trois mois en présentiel et deux mois en semi-présentiel. Pour les C1S4, C2S4, C3S4 et C4S4, il est prévu un mois en présentiel et quatre mois en semi-présentiel.

La configuration de la plateforme Moodle de cours en ligne (plateforme d'apprentissage en ligne LMS (Learning Management System) associe un Système de Gestion de Contenu (SGC), assurant la gestion des ressources pédagogiques du cours, des activités d'apprentissage interactives (fonctions pédagogiques d'évaluation, de communication ou/et de collaboration). La plateforme offre la possibilité aux enseignant-es et aux étudiant-es d'interagir en utilisant les fonctions « activités » ou « ressources » pour réaliser des tâches en lien avec le cours.

\subsection{Une réponse, de nouvelles possibilités d'apprentissage}

Créé pour répondre à des besoins cruciaux en ressources humaines pour développer et gérer des projets-programmes éducatifs dans le domaine des alternatives éducatives, le M-ICGAE a permis à certains étudiant-es professionnel-les, qui ne peuvent pas suivre les cours à temps plein à Niamey au Niger, de rester dans leurs pays respectifs sur les périodes des cours à distance. Les étudiant-es voient ainsi les coûts élevés liés aux déplacements (transport, hébergement, nourriture, assurance santé) baissés pour les étudiant-es basés hors du Niger.

Le M-ICGAE a été l'occasion d'identifier les besoins de formations des acteurs en alternatives éducatives, faire l'état des lieux des offres de formation de l'UAM et envisager un accompagnement pour renforcer certaines offres et en proposer de nouvelles si nécessaire, de manière régulière, avec une programmation connue d'avance (maquette de formation).

Le Master a été également mis en contact des universitaires avec les milieux professionnels pour travailler sur la méthodologie d'élaboration des programmes de formation

\footnotetext{
${ }^{5} \mathrm{C} 1 \mathrm{~S} 1$ (sept.) signifie première cohorte, premier semestre, semestre mois de démarrage du semestre
} 
professionnalisants, afin de permettre aux Facultés d'identifier des filières de formation professionnalisantes à développer prioritairement à l'UAM.

\section{CONCLUSION}

L'enseignement à distance est une ambition grandement affichée par l'UAM pour ses apports supposés réels dans les programmes d'enseignement et de formation. Cette ambition s'est inscrite dans un processus de réflexion et de questionnement sur le développement de l'éducation numérique et sur comment accompagner les enseignant-es pour développer des espaces d'apprentissage et de formation.

Nous savons que l'enseignement à distance est un dispositif innovant qui n'est pas très bien développé dans les pratiques universitaires. II reste au stade des intentions et peine souvent à se développer comme solution pour enseigner et apprendre autrement. II fait l'objet d'une attention particulière, mais les réflexions se concentrent autour de son ancrage comme une alternative aux nombreux cours en présentiel.

Nous avons montré les possibilités offertes par l'enseignement à distance. Celles-ci nécessitent des recadrages dans l'organisation des enseignements-apprentissages et un accompagnement technico-pédagogique des enseignant-es et des étudiant-es (Ferone, 2012). Dans les pratiques universitaires d'enseignement-apprentissage, il est important de bien penser les logiques d'enseignement pour situer la place et le rôle que peut jouer l'enseignement à distance.

L'efficacité de cette modalité de formation à distance, permet de « créer de la présence à distance » (Jézégou, 2010). Mais il est important avant tout de cerner et identifier les besoins des cibles, identifier et analyser les opportunités à saisir, ainsi que les problèmes susceptibles d'être des obstacles au développement de l'enseignement à distance. L'exemple du Master que nous avons présenté montre bien que, l'enseignement à distance peut être une initiative porteuse pour les apprenant-es, si le dispositif est bien pensé et que la connexion Internet est stable.

\section{RÉFÉRENCES}

Akkari, A., \& Soumana, H. (2014). Évaluation externe du Programme Régional Interinstitutionnel pour la Qualité de l'Éducation par la Pédagogie du Texte (PRIQUE/PdT). Berne : DDC.

Akouété-Hounsinou, F. (2012). La formation continue à distance des enseignants du secondaire au bénin : réalités et perspectives. Thèse de Doctorat en sciences de l'éducation, Université de Montréal, Canada. https://papyrus.bib.umontreal.ca/xmlui/handle/1866/9003 
Attenoukon, S. A. (2011). TIC et rendement académique en contexte universitaire béninois : cas des apprenants en droit de l'Université d'Abomey-Calavi. Thèse de Doctorat en sciences de l'éducation, Université de Montréal, Canada. http://hdl.handle.net/1866/5139

Attenoukon, S. A. (2020). Comment les enseignants et apprenants universitaires au Bénin utilisent-ils les technologies ? In T. Karsenti, K. Toure, M. Lepage, \& S. A. Attenoukon, Usages et appropriation des technologies éducatives en Afrique : Quelques pistes de réflexion (p. 191-216). Bamenda : Langaa.

Bruillard É. (2010). Formation à distance : Dispositifs techniques. Structuration organisationnelle, spatiale et temporelle des environnements. Distances et Savoirs, 8(2), 207-221.

Chan, C., Embi, M., \& Hashim, H. (2019). Primary school teachers' readiness towards heutagogy and peeragogy. Asian Education Studies, 4(1), 11-21. http://dx.doi.org/10.20849/aes.v4i1.602

Ferone, G. (2011). Interagir à distance en formation des maîtres. Recherche et Formation, 68, 79-94.

Ferone, G. (2012, 6-8 septembre). S'engager dans la formation des maîtres à distance. Un paradoxe ? JOCAIR (Journées communication et apprentissage instruments en réseau), Amiens.

Ferone, G., \& Lavenka, A. (2019). La classe virtuelle, quels effets sur la pratique de l'enseignant? Distances et Médiations des Savoirs, 10. https://journals.openedition.org/dms/1047\#quotation

Jezegou A. (2008). Apprentissage autodirigé et formation à distance. Distances et Savoirs, 8, 343-364.

Jezegou A. (2010). Créer de la présence à distance en e-learning : Cadre théorique, définition, et dimensions clés. Distances et Savoirs, 8(2), 257-274.

Karsenti, T. (2009). Intégration pédagogique des TIC : Stratégies d'action et pistes de réflexion. Ottawa : CRDI.

Mohamed Sagayar, M. (2018). Les ressources numériques : Analyse des pratiques, représentations, besoins et attentes des usagers dans les universités publiques du Niger. Réseau Africain Francophone d'Education Comparée (RAFEC), 5, 309-333.

Murphy, P., Anzalone, S., Bosch, A., \& Moulton, J. (2002). Améliorer les possibilités d'apprentissage en Afrique : L'enseignement à distance et les technologies de l'information et de la communication au service de l'apprentissage. Washington, DC : Banque mondiale. http://documents1.worldbank.org/curated/en/745721468003017732/pdf/240510FRENC HOameliorer.pdf

Ouedraogo, B. (2020). Les professeurs des universités au Burkina Faso embrassent-ils les technologies éducatives ? In T. Karsenti, K. Toure, M. Lepage, \& S. A. Attenoukon, Usages et appropriation des technologies éducatives en Afrique : Quelques pistes de réflexion (pp. 241-288). Bamenda : Langaa.

Power, M. (2002). Générations d'enseignement à distance, technologies éducatives et médiatisation de l'enseignement supérieur. Revue de l'Éducation à Distance, 17(2), 57-69.

Sensevy, G. (2018). L'action conjointe en didactique. Animation \& Éducation, 265, 48-50. 
UNESCO. (2016). Déclaration d'Incheon et cadre d'action pour la mise en œuvre de l'objectif de développement durable 4. Paris : UNESCO.

Open Access Publications - Bibliothèque de l'Université de Genève Creative Commons Licence 4.0

(c) (1) 